\title{
Production of Hyperpolarized ${ }^{3} \mathrm{He}$ Gas for Medical Imaging
}

\author{
Masayoshi Tanaka \\ Kobe Tokiwa Univ. \\ E-mail: tanaka@rcnp.osaka-u.ac.jp
}

Seiji Makino ${ }^{a}$, Hisako Fujimura $^{a}$, Takeshi Ohta ${ }^{b}$, Masaru Yosoi $^{h}$, Mamoru Fujiwara $^{b}$, Yuto Kasamatsu ${ }^{b}$, Kunihiro Ueda ${ }^{c}$, Gérard Rouilléd ${ }^{d}$, Giorgio Frossati ${ }^{e}$, and A. de Waard ${ }^{e}$

${ }^{a}$ Department of Physics, Wakayama Medical University, 580 Mikuzu, 641-001/ Wakayama, Japan

${ }^{b}$ Research Center for Nuclear Physics, Osaka University, Mihogaoka 10-1, Ibaraki, Osaka 567-0047, Japan

'Department of Clinical Technology, Kohe Tokiwa Lniversity; Ohtani-cho 2-6-2, Nagata, Kobe 653-0838, Japathe $n$

"Imagerie par Résonance Magnétique Médicale et Multi-Modalités, UMR 8081 Université Paris

Sud 11 -Bâtiment Rue André Ampère 91406, Orsay, Cedcx France

"Leiden Cryogenics BV. Kenawweg 112331 BA Leiden. The Netherlands

We present the latest development on the hyperpolarized ${ }^{3} \mathrm{He}$ for MRI (Magnetic Resonance Imaging) produced by the brute force and rapid melting method which use the Pomeranchuk cooling under the strong magnetic field $(\sim 17 \mathrm{~T})$ and low temperature $(\sim 10 \mathrm{mK})$. To improve vulnerability of the piston and bellows type Pomeranchuk cell, we designed a plastic (PEI) type Pomeranchuk cell with a capton membrane to reliably propagate ${ }^{4} \mathrm{He}$ gas pressure to ${ }^{3} \mathrm{He}$. We also improved a thermal conductivity between ${ }^{3} \mathrm{He}$ and a high purity copper cold finger connected to the mixing chamber of the ${ }^{3} \mathrm{He} /{ }^{4} \mathrm{He}$ dilution refrigerator (DRS2500) by using a sintered silver rod. A temperature sensor and a sapphire pressure gauge were positioned inside the ${ }^{3} \mathrm{He}$ space to increase response and sensitivity. For a preliminary test before main experiments with the DRS2500, we introduced a cryofree dilution refrigerator for overall system check.

Secondly, a newly commenced project of the ${ }^{19} \mathrm{~F}$ in PFC (PerFluoroCarbon) is presented. Here, PFC has been well established as an artificial blood. The PHIP (ParaHydrogen Induced Polarization) method is getting ready for production of the hyperpolarized ${ }^{19} \mathrm{~F}$ in PFC. Since the PHIP uses hydrogenation of para-hydrogen $\left(\mathrm{p}-\mathrm{H}_{2}\right)$ as medium to generate the nuclear polarization, we currently make an effort in producing $\mathrm{p}-\mathrm{H}_{2}$ by using a refrigerator and a catalyst consisting of paramagnetic compounds.

XVIth International Workshop in Polarized Sources, Targets, and Polarimetry

14-18 September 2015

Ruhr-University Bochum, Germany

* Speaker.

${ }^{\dagger}$ A footnote may follow. 


\section{Introduction}

It is well-known that Japanese radiation doses are exceptionally high due to the radiations, $\mathrm{X}$-rays, and $\gamma$-rays for medical diagnosis [1]. Therefore, we are seriously aware of the radiation injury. The national urgent subject is how to reduce the radiation exposure. Motivated by the above requirement, we chose the MRI with the hyperpolarized nuclei as one of the novel diagnostic methods free from radiation.

Meanwhile, polarized ion sources and targets for nuclear physics research have been developed at RCNP, Osaka University for many years. Owing to our experience on the nuclear spin handling accumulated so far, we could start a project to produce hyperpolarized ${ }^{3} \mathrm{He}$ gas [2] as a contrast medium of MRI (Magnetic Resonance Imaging) for lung imaging, where 'hyperpolarized' signifies that polarized higher than the thermal cquilibrium values popularly used at clinics and hospitals. A few years later, we further started another project, i.e., production of hyperpolarized ${ }^{19} \mathrm{~F}$ in PFC (PerFluoro Carbon) [3] as a constrast medium of MRI for diagnosis of the disease associated with cardiovascular system.

In this report, we present the status quo of the development for the ${ }^{3} \mathrm{He}$ and ${ }^{19} \mathrm{~F}$ hyperpolarization, respectively, and discuss the future strategy as an extention of the development. In addition, one of our colleagues discusses, in this workshop, a new experimental result on the high frequency digital NMR spectrometer required not only for our project (hyperpolarized MRI) but also for the polarized HD project on going at SPring- $\$[4,5]$.

\section{Status quo}

\subsection{Production of Hyperpolarized ${ }^{3} \mathrm{He}$}

An idca to produce hyperpolarized liquid ${ }^{3} \mathrm{He}$ by the rapid melting method was proposed firstly by Castaing et al. [6] for studying the fundamental physics of the liquid ${ }^{3} \mathrm{He}$, and experimentally verified by the group from Leiden later [7].

Encouraged by this success, Frossati proposed a deliberate plan to produce a hyperpolarized ${ }^{3} \mathrm{He}$ gas as an extention of the Castaing method. The idea of Frossati is the formation of hyperpolarized solid ${ }^{3} \mathrm{He}$ by compressing liquid ${ }^{3} \mathrm{He}$ with a Pomeranchuk cell under an external field of $15 \mathrm{~T}$, and subsequent rapid melting and gasification of hyperpolarized solid ${ }^{3} \mathrm{He}$ shorter than spin relaxation times in each ${ }^{3} \mathrm{He}$ phases (solid, liquid and gas) [8].

Guided by this scenario, the RCNP group developed to produce hyperpolarized ${ }^{3} \mathrm{He}$ by the Pomeranchuk cooling under the magnetic field of $17 \mathrm{~T}$ and temperature around $10 \mathrm{mK}[2$, 3]. The gas pressure of the Pomeranchuk cell was controlled by a combination of piston and bellows. To realize the temperature lower than $10 \mathrm{mK}$, the ${ }^{3} \mathrm{He} /{ }^{4} \mathrm{He}$ dilution refrigerator, DRS2500 (Leiden Cryogenics) which has a cooling power of $2500 \mu \mathrm{W}$ at $100 \mathrm{mK}$ was employed. To generate the homogencous magnetic ficld of $17 \mathrm{~T}$, the superconducting solenoid, 17T-71 (JASTEC) was employed.

After a number of experiments with the afore-mentioned system, it was found that the performance of the piston type Pomeranchuk cell often got many technical troubles associated with failures of mechanics and thermodynamics. To overcome this difficulty, we improved the Pomeranchuk cell by replacing it with a plastic (PEI) cell with a capton (DuPont-Toray) membrane as a 
pressure transmitter from ${ }^{4} \mathrm{He}$ to ${ }^{3} \mathrm{He}$. To improve thermal contact by reducing the Kapitsa resistance, we used a sintered silver rod. For monitoring the ${ }^{3} \mathrm{He}$ pressure and temperature inside the ${ }^{3} \mathrm{He}$ cell, whose measurements were very important to control the Pomeranchuk cooling precisely, a sapphire pressure gauge [9] and a carbon resistance available on the commercial base were used, respectively.

The new Pomeranchuk ecll thus designed is illustrated in Fig. 1-(a), and photos attached to the DRS2500 through a high purity cold finger are shown in Fig. 1-(b) and Fig. 1-(c), respectively.

(a)

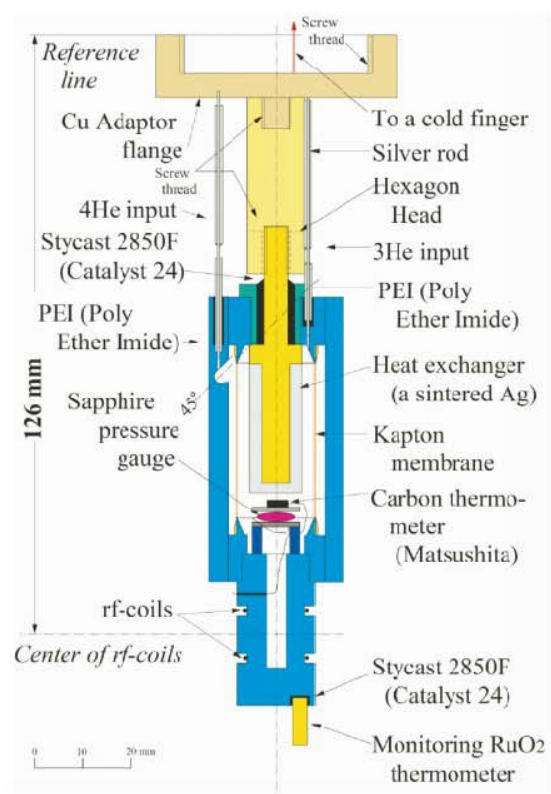

(b)

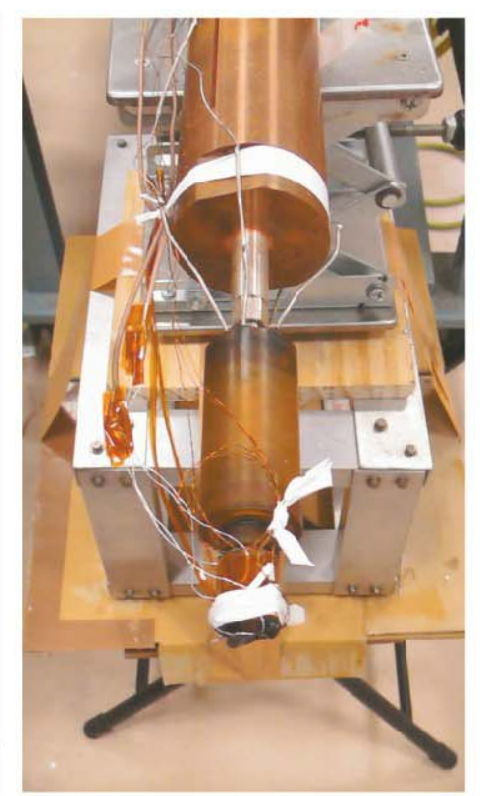

(c)

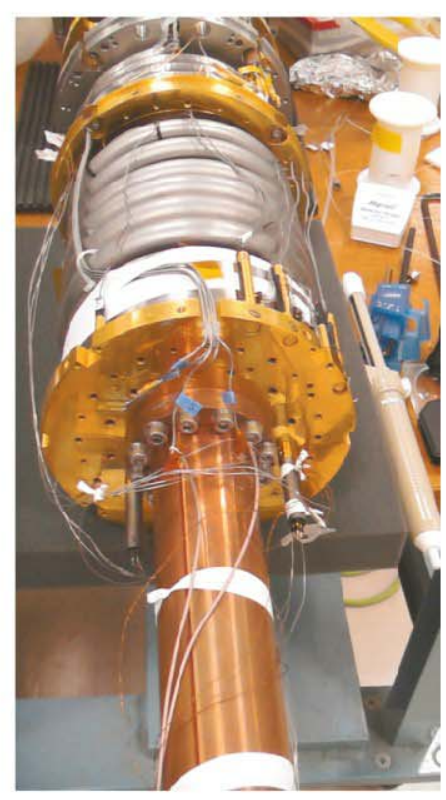

Figure 1: (a) Drawing of a new plastic Pomeranchuk cell, where the ${ }^{3} \mathrm{He}$ gas pressure is controlled through the capton membrane by ${ }^{4} \mathrm{He}$ gas. (b)The Pomeranchuk cell is altached to a high pury $\mathrm{Cu}$ cold finger. (c)The high purity $\mathrm{Cu}$ cold finger is connected to a mixing chamber plate of the DRS2500.

It is of particular importance to make a test experiment before the main experiment with the DRS starts. In fact, we had, in a series of the previous work, many troubles unexpectedly. In order to avoid further consumption of useless time, it is an important step to use another dilution refrigerator easily handled. For this purpose, we introduced a cryofree ${ }^{3} \mathrm{He} /{ }^{4} \mathrm{He}$ dilution refrigerator $(\mathrm{KOBE} 10 \mu) \mathrm{A}$ drawing of KOBE10 $\mu$ is shown in Fig. 2-(a). Photos of the inner side and the whole layout of the system are shown in Fig. 2-(b) and Fig. 2-(c), respectively. The performance of KOBE $10 \mu$ has a cooling power down to $100 \mathrm{mK}$, which is large enough for the test experiment.

Another improvement was successful observation of the proton NMR signals at $17 \mathrm{~T}$ by using a digital NMR spectrometer employing the PXI modules working at GHz regions. Since our previous NMR spectrometer was dedicated to the NMR signals working at $1 \mathrm{~T}$ [4], this new NMR spectrometer will hopefully be a direct polarization monitor at $17 \mathrm{~T}$. The detailed description is presented in this workshop by one of our group [5]. 
(a)

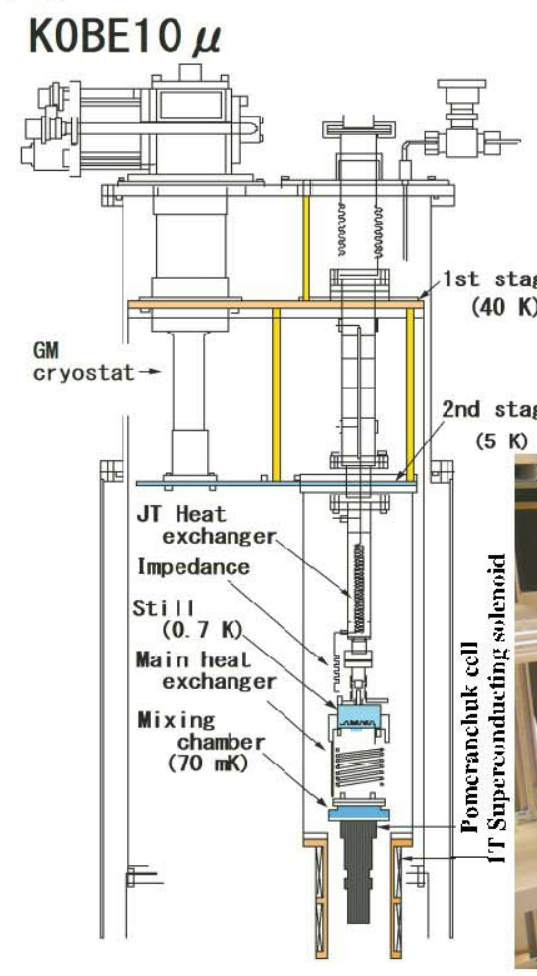

(b)

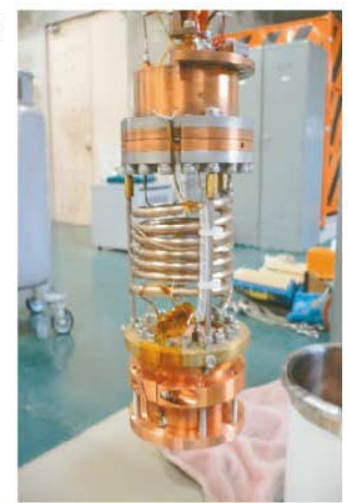

(c)

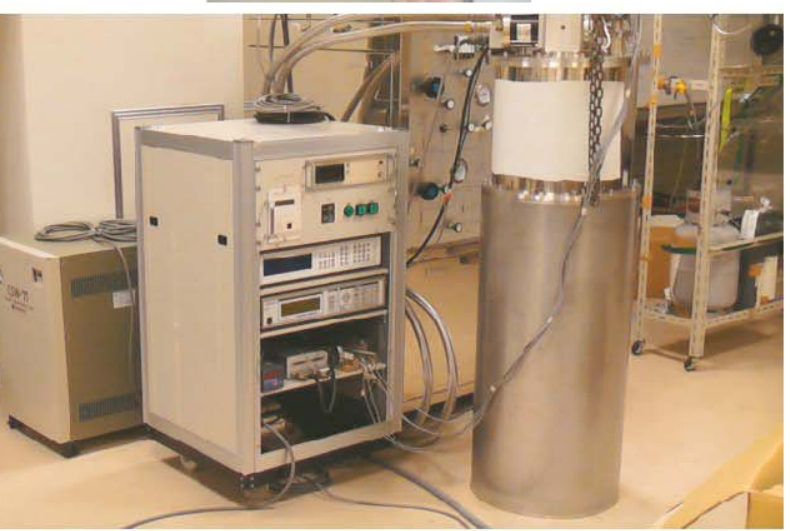

Figure 2: (a) Drawing of a cryofree ${ }^{3} \mathrm{He} /{ }^{4} \mathrm{He}$ dilution refrigerator $(\mathrm{KOBE} 10 \mu)$. (b) Picture of Cryofree ${ }^{3} \mathrm{He} /{ }^{4} \mathrm{He}$ dilution refrigerator. (c) Picture of a whole system of the KOBE $10 \mu$.

\subsection{Production of Hyperpolarized ${ }^{19} \mathrm{~F}$}

Since a short description on the motivation and principle of the PHIP method applied to hyperpolarization of ${ }^{19} \mathrm{~F}$ in PFC (PerFluoroCarbon) is given in Ref. [3], the description in this report is restricted to the development in production of parahydrogen $\left(\mathrm{p}-\mathrm{H}_{2}\right)$ by means of low temperature and catalyst.

To hyperpolarize ${ }^{19} \mathrm{~F}$ in PFC by the PHIP method [3], we must prepare $\mathrm{p}-\mathrm{H}_{2}$ whose proton spins are antiparallel each other. As shown in Fig. 3-(a), concentration of $\mathrm{p}-\mathrm{H}_{2}$ is only $25 \%$ at room temperature. However, the $\mathrm{p}-\mathrm{H}_{2}$ concentration is increased according as temperature decreases due to the molecular energy difference between $\mathrm{p}$ - and ortho-hydrogen $\left(\mathrm{o}-\mathrm{H}_{2}\right)$. Note that at lower temperature than $20 \mathrm{~K}$, most of $\mathrm{o}-\mathrm{H}_{2}$ converts to $\mathrm{p}-\mathrm{H}_{2}$.

To realize this, a temperature of purified $\mathrm{H}_{2}$ gas was cooled down to $\mathrm{T}=22 \mathrm{~K}$ with a gas pressure of 0.3 to $1 \mathrm{~atm}$. under the presence of paramagnetic compound, $\mathrm{FeO}(\mathrm{OH})$ (Sigma Aldrich), where the paramagnetic compound acts as a catalyst to accerelate the transition from $\mathrm{o}-\mathrm{H}_{2}$ to $\mathrm{p}$ $\mathrm{H}_{2}[10]$.

A gas chromatography was used for the measurement of the contents of $\mathrm{p}-\mathrm{H}_{2}$ and $\mathrm{o}-\mathrm{H}_{2}$ [11]. One of observed spectra for partial pressures for $\mathrm{p}-\mathrm{H}_{2}$ and $\mathrm{o}-\mathrm{H}_{2}$ is shown in Fig. 3-(b). It was found that the major peak was due to p-hydrogen with less pronounced $\mathrm{o}-\mathrm{H}_{2}$ peak at $22 \mathrm{~K}$, which is enough for our future PHIP project. More detailed description of the content of $\mathrm{p}-\mathrm{H}_{2}$ will be 
published elsewhere.

(a)

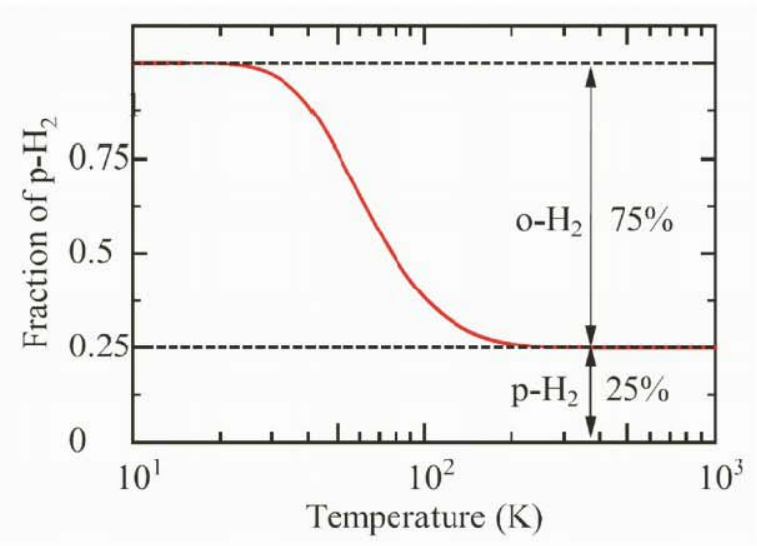

(b)

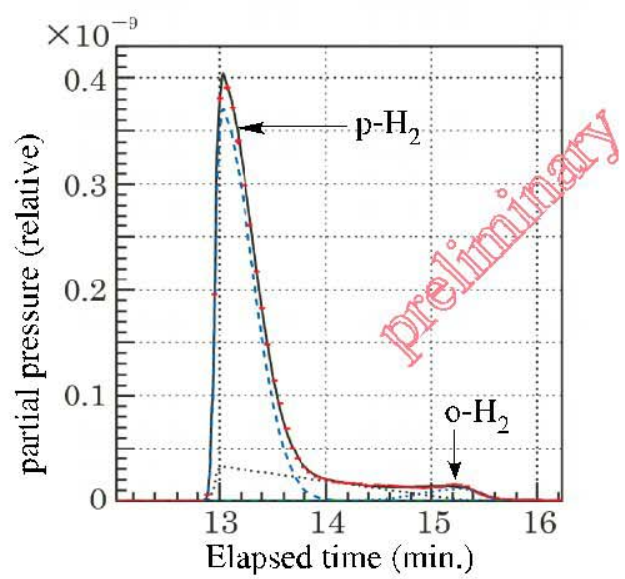

Figure 3: (a) Calculated concentrations for $\mathrm{p}-\mathrm{H}_{2}$ and $\mathrm{o}-\mathrm{H}_{2}$ plotted as a function of temperature. (b) Observed partial pressures (relative values) of $\mathrm{p}-\mathrm{H}_{2}$ and $\mathrm{p}-\mathrm{H}_{2}$ measured as an elapsed time by gas chromatography. The peaks at 13.0 and $15.2 \mathrm{~min}$. correspond to $\mathrm{p}-\mathrm{H}_{2}$ and $\mathrm{o}-\mathrm{H}_{2}$, respectively. Here, the column of the gas chromatography is a varian CP Molesive, the carrier gas is helium, the gas temperature is $110-120 \mathrm{~K}$, and flow rate is $1.0 \mathrm{~m} \ell / \mathrm{min}$.

\section{Conclusion and future prospect}

The status quo of the hyperpolarization project on ${ }^{3} \mathrm{He}$ and ${ }^{19} \mathrm{~F}$ is not so well progressive due to our unaccustomed skill. We recently introduced a cryofree dilution refrigerator with which we will conduct a preliminary series of test experiments for the new plastic Pomeranchuk cell system before recommencing the main expcriment, thus hopefully enabling us to speed up the hyperpolarized ${ }^{3} \mathrm{He}$ project. The hyperpolarized ${ }^{19} \mathrm{~F}$ project is also awaiting the search for the hydrogenation catalysts suitable for the PHIP.

As one of promising future hyperpolarized MRI the following two experimental breakthroughs recently reported should be reminded: The first one is that the hyperpolarization of other nuclei such as ${ }^{13} \mathrm{C}$ could be made with a more downscaled refrigerator [12] than the expensive ones requesting a high cooling power.

The second breakthrough is the recent success by the US group [13]. They observed position and time spectra of the hyperpolarized ${ }^{13} \mathrm{C}$ in lactate quickly $(\sim 10 \mathrm{sec}$.) formed after the metabolic reaction with pyruvic acid labeled with the hyperpolarized ${ }^{13} \mathrm{C}$ accumulated in the prostate cancer cells by injection into a vein. Though it has been long considered that the hyperpolarized nuclei could not be applied to the cancer MRI which needs a time longer than, e.g., an hour since the spin relaxation times of hyperpolarized nuclei are far shorter than a minute. The success this time may hopefully enable us to easily apply the hyperpolarized MRI to medical diagnoses even to the cancer MRI. 


\section{References}

[1] A. B. Gonzalez, and S. Darby, Lancet 363, 345-351 (2004).

[2] M. Tanaka, T. Kunimatsu, M. Fujiwara, H. Kohri, T. Ohta, M. Utsuro, M. Yosoi, S. Ono, K. Fukuda, K. Takamatsu, K. Ueda, J. -P. Didélez, G. Frossati and A. de Waard, J. Conference Series 295 (SPIN2010), 012167, (2011).

[3] M. Tanaka, H. Kohri, T. Ohta, M. Yosoi, M. Fujiwara, K. Ueda, S. Imoto, K. Takamatsu, J. -P. Didélez, G. Frossati, A. de Waard, Yu. Kiselev, S. Makino, H. Fujimura, K. Fukushima, S. Fukushima, and H. Kondoh, Physics of Particles and Nuclei, 44959 (2013).

[4] T. Ohta, M. Fujiwara, K. Fukuda, H. Kohri, T. Kunimatsu, C. Morisaki, S. Ono, M. Tanaka, K. Ueda, M. Uraki, M. Utsuro, S. Y. Wang, M. Yosoi, Nucl. Instr. and Meth. A633 46 (2011).

[5] Takeshi Ohta, Hideki Kohri, Masaru Yosoi, Mamoru Fujiwara, Masayoshi Tanaka, 16th International Workshop in Polarized Sources, Targets, and Polarimetry, 2015, PSTP2015, Bochum, Germany.

[6] B. Castaing, and P. Noziéeres, J. Phys., 40257 (1979).

[7] G. A. Vermeulen, S. A. J. Wiegers, C. C. Kranenburg, R. Jochemsen, and G. Frossati, Canadian J. Phys. 65560 (1987).

[8] G. Frossati, Nucl. Instr. and Mcth.,A 402479 (1998).

[9] W. Griffioen, and G. Frossati, Rev. Sci. Instr. 561236 (1985).

[10] K. Mishima, M. Utsuro, Y. Nagai, M. Tanaka, T. Kohmoto, Y. Fukuda, Y. Kiyanagi, and M. Ooi, Phys. Rev. B 75014112 (2007).

[11] T. Ohta, S. Bouchigny, J.-P. Didelez, M. Fujiwara, K. Fukuda, H. Kohri, T. Kunimatsu, C. Morisaki, S. Ono, G. Rouillé, M. Tanaka, K. Ueda, M. Uraki, M. Utsuro, S. Y. Wang, and M. Yosoi, Nucl. Instr. Meth. A 664 347-352 (2012).

[12] M. L. Hirsch, N.Kalechofsky, A. Belzer, M. Rosay, and J. G. Kempf, J. American Chemical Socicty, 1378428 (2015).

[13] S. J. Nelson, J. Kurhanewicz, D. B. Vigneron, P. E. Z. Larson, A. L. Harzstark, M. Ferrone, M. van Criekinge, J. W. Chang, R. Bok, I. Park, G. Reed, L. Carvajal, E. J. Small, P. Munster, V. K. Weinberg, J. H. Ardenkjaer-Larsen, A. P. Chen, R. E. Hurd, Liv-Ingrid Odegarstuen, F. J. Robb, J. Tropp, and J. A. Murray, Science Translational Medicine 5 198ra108- 1 - -10 (2013). 